\title{
Optoelectronics System for Estimating Measurement Error of Image Pattern Correlation Technique
}

\author{
A.Yu. Poroykov ${ }^{1}$, T. Kirmse ${ }^{2}$, F. Boden ${ }^{2}$, K.M. Lapitskiy ${ }^{1}$, Yu.V. Ivanova ${ }^{1}$, I.A. Lapitskaya ${ }^{1}$ \\ ${ }^{1}$ National Research University "Moscow Power Engineering Institute"(MPEI), Krasnokazarmennaya \\ 14, Moscow, 111250 Russia, \\ ${ }^{2}$ German Aerospace Center (DLR), Bunsenstraße 10, 37073 Göttingen, Germany \\ poroykovay@gmail.com
}

\begin{abstract}
:
Photogrammetric methods for in-flight deformation measurements have undeniable advantages: e.g. contactless measurements, measuring the entire surface and the relative simplicity of the setup. During the European research projects Advanced In-Flight Measurement Techniques (AIM and AIM2) an implementation of the photogrammetric method - Image Pattern Correlation Technique (IPCT) has been proposed. It had been successfully applied in several flight test campaigns.

To evaluate the measurement error of photogrammetric methods either opto-geometric evaluations or digital modeling of synthetic images are commonly used. Some works indicate only error estimation, but without the explanation of its origin. The paper presents an approach for estimating the measurement error for the IPCT. An experimental system is developed including an imitator of the surface deformation and an additional optical sensor for measuring the present surface profile. The error is determined as a difference between the results of the optical sensor and the IPCT. The system allows to simulate a deformation with an amplitude of $\pm 25 \mathrm{~mm}$ on a surface area of $380 \times 380 \mathrm{~mm}^{2}$ in increments of $0.5 \mathrm{~mm}$ horizontally and $0.001 \mathrm{~mm}$ vertically, with a measurement accuracy of $0.075 \mathrm{~mm}$. The system will be outlined in the paper and measurement results are presented.
\end{abstract}

Key words: optoelectronics system, deformation measurement, accuracy estimation, photogrammetry, IPCT.

\section{Introduction}

The problem of surface deformation measurement occurs in many branches of science and technology. It can be found in the automotive industry, construction and aviation.

Different methods can be used to solve this problem. All of them can be conditionally divided into three groups: methods using straingauge, piezoelectric and mechanical sensors, optical and photogrammetric methods.

The principle of operation of the first group of methods is based on effects like tensoresistive effect, direct piezoelectric effect and others. To measure deformations surface-distributed sensor arrays can be used. Disadvantages of such methods are the locality of the measurements, direct contact of the sensing element with the test sample, the need for calibrating the sensors, and the influence of environmental factors on the measurement results.
The methods of the second group - optical methods, have an important advantage over the methods of the first - the are non-intrusive. The second group includes many different optical methods: the grid method [1], the moiré method [2], the photoelasticity method [3], the method of holographic and speckle-interferometry [4-5]. The shortcomings of optical methods include the need to make exact copies of the test samples, the complexity of processing the obtained results, and the need to use complex optical systems.

So for in-flight measurements most methods of the first and the second group involve a large installation effort. The third group photogrammetric methods can be partially attributed to optical methods. However, it is based on digital signal processing with minimal use of optical systems.

The Image Pattern Correlation Technique (IPCT) is a modern implementation of the photogrammetric methods for measuring deformations based on digital image processing 
[6-8]. This non-intrusive method captivates by its simple experimental setup. It requires two digital cameras, a correlation pattern on the surface to be measured and a computer for image acquisition and processing using modern image processing algorithms. Therefore, this method is well suited for the use in field experiments such as in-flight measurements.

To determine the deformation of the object a special correlation pattern is applied to the investigated areas. Generally, the pattern consists of randomly placed dots. Images of pattern are obtained with digital cameras before and after deformation. Further cross-correlation processing of the registered images allows to calculate the displacement field of the pattern. This field can be used to reconstruct the deformed surface profile by means of triangulation.

Although the method itself is relatively easy to implement, the analysis of its measurement error is difficult because of the multiple parameters which influences the accuracy of the method like the calibration of the camera and the use of cross-correlation to determine point correspondences in the stereo views. Therefore, it is difficult to estimate the error using direct calculations. In general, either optogeometric evaluations [9-13], or digital modeling of synthetic images [14-15] are commonly used for its estimation. However, these approaches cannot take into account all the factors that arise in real experiments. This problem can be solved by comparing the results of IPCT measurements with the results of measurements obtained by another reference method. This approach will allow to take into account all the factors influencing the error, and to estimate it within the frame of the accuracy of the reference method.

\section{Measurement Techniques}

For the automation of measurements, a software and hardware optoelectronics system was created to estimate the IPCT measurement error by MPEl. It is based on the simple idea to use a surface whose deformation occurs in a given way (an imitator of a deformable surface) and a high-precision optical sensor which can precisely measure surface deformation. Comparing the results obtained by sensor and by IPCT we can estimate the absolute error of the photogrammetric measurements within the scope of the error of the optical sensor.

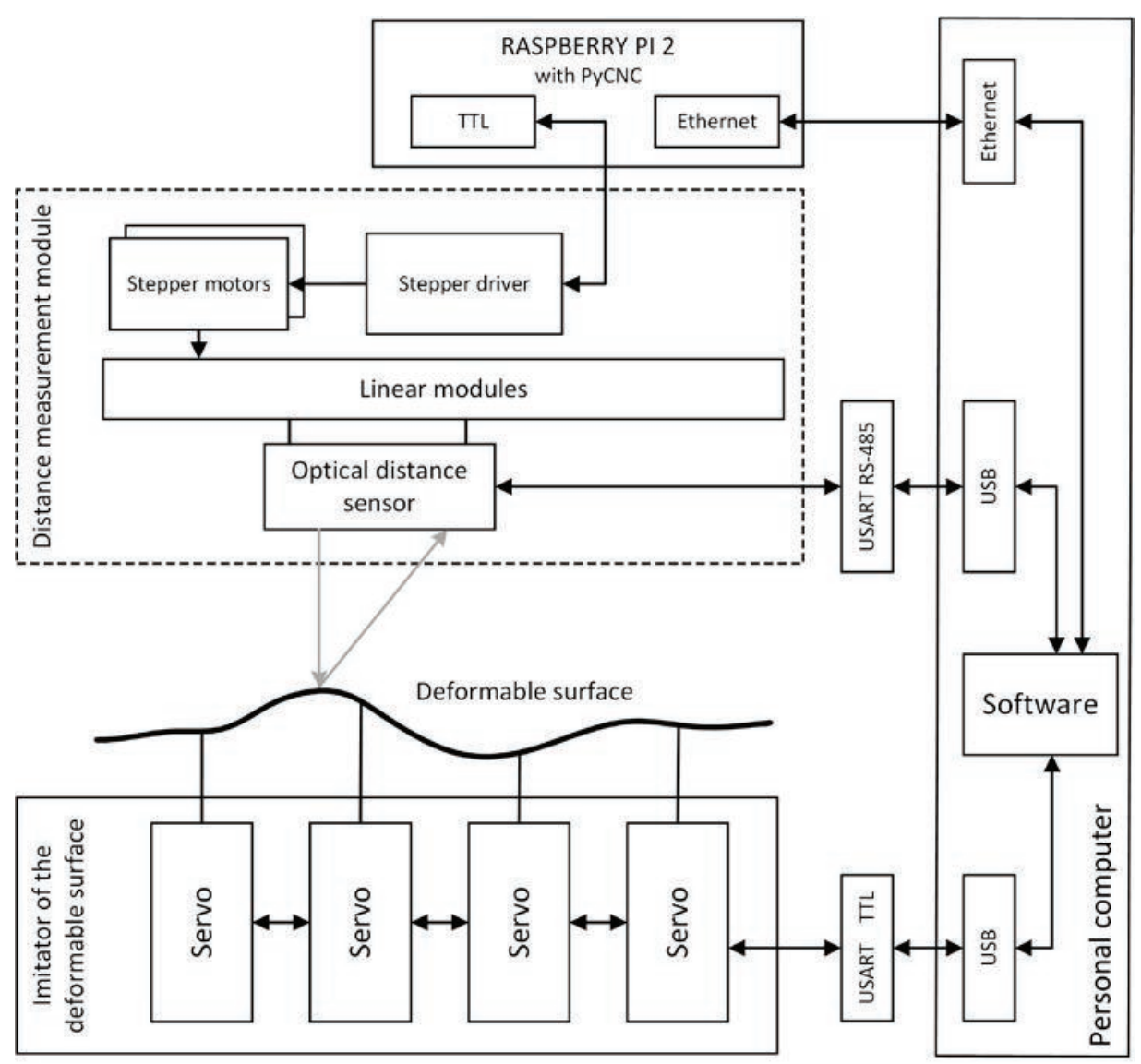

Fig. 1. The functional scheme of the optoelectronics system for estimating IPCT measurement error 
To measure the entire surface by one sensor, linear displacement modules with stepper motors were used. The sensor measures the distance to the surface at a grid of points of a defined distance by moving above the surface in two perpendicular directions. Stepper motors allow to control the position of the sensor with increments of $0.1 \mathrm{~mm}$ and error no more than $0.5 \mathrm{~mm}$.

The proposed system consists of three main components: an imitator of a deformable surface, a module for measuring the distance to the surface and software for a personal computer. The functional scheme of the system is shown in Fig 1.

The imitator of the deformable surface consists of an aluminum base and several servos fixed on it. Each servo is rigidly connected to a section of a flexible plate located above the servos. The plate acts as a deformable surface with an area of $380 \times 380 \mathrm{~mm}^{2}$. The total number of servos is 16 . They are evenly distributed under the plate. Changing the position of the servo arm leads to a proportional displacement of the plate part above it in the vertical direction. The amplitude of the displacements for each servo is $\pm 25 \mathrm{~mm}$. Appearance of the imitator of the deformable surface is shown in Fig. 2.

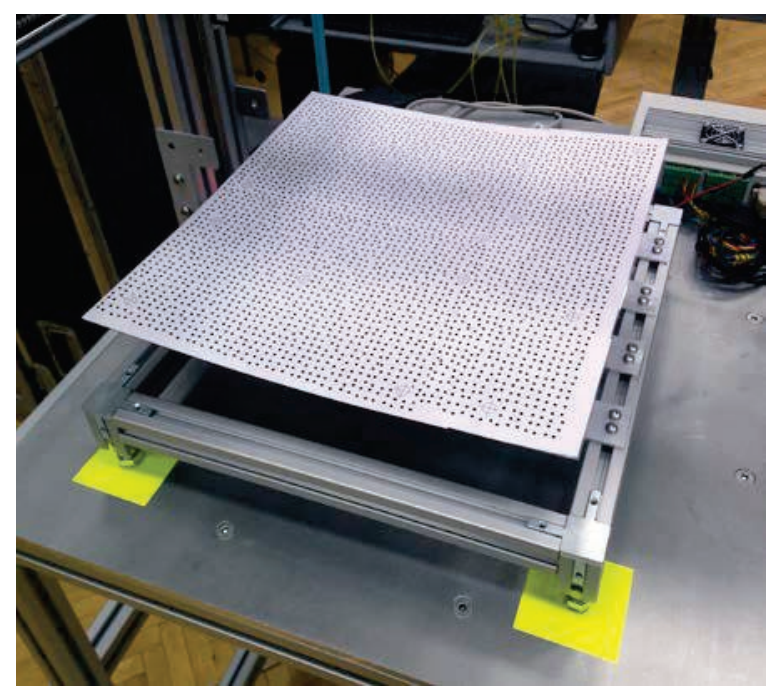

Fig. 2. Appearance of the imitator of the deformable surface

The module for measuring the distance to the surface consists of two linear displacement modules perpendicular to one another and optical sensor. Their lengths are $500 \mathrm{~mm}$ for $X$ direction and $400 \mathrm{~mm}$ for $\mathrm{Y}$ direction. The optical sensor LS5-40/50 "NPP PRIZMA" measures distance based on the triangulation of the laser beam reflected from the measured surface. Sensor has a resolution of $0.001 \mathrm{~mm}$ and an error of not worse than $0.075 \mathrm{~mm}$ in the range of distances of $50 \mathrm{~mm}$ and the minimal distance to the sensor of $40 \mathrm{~mm}$. The spot size of the laser beam of the sensor in the middle of the measurement range is $175 \mu \mathrm{m}$ and does not exceed $300 \mu \mathrm{m}$ in the entire range.

The software part is designed to control the entire installation as a whole: setting the positions of the servos (deformation of the object) and displaying the results of the distance measuring unit to the surface using a graphical interface.

The software part of the test setup was developed in $\mathrm{C \#}$ and Python language for a personal computer. The program allows to set the positions of each servo separately and displays the distances to the surface measured by the sensor.

\section{System Distance Measurement Accuracy}

An experimental investigation was carried out to estimate the accuracy and repeatability of the optical sensor data for the complete measurement volume. For this purpose we used a granite surface plate with grade 00 , which provides non-flatness no more then $3 \mu \mathrm{m}$ on its area of $400 \times 400 \mathrm{~mm}^{2}$.

The method of investigation consisted in the multiple measurement of the same points on the surface of the plate at different moments of time. 400 points were uniformly distributed over the entire surface of the plate. Every day from 5 to 20 measurements of the entire surface of the plate were carried out. For all measurements for one day, the average deviation from the mean for each point was calculated, and then they were averaged. The obtained data is presented in Fig. 3.

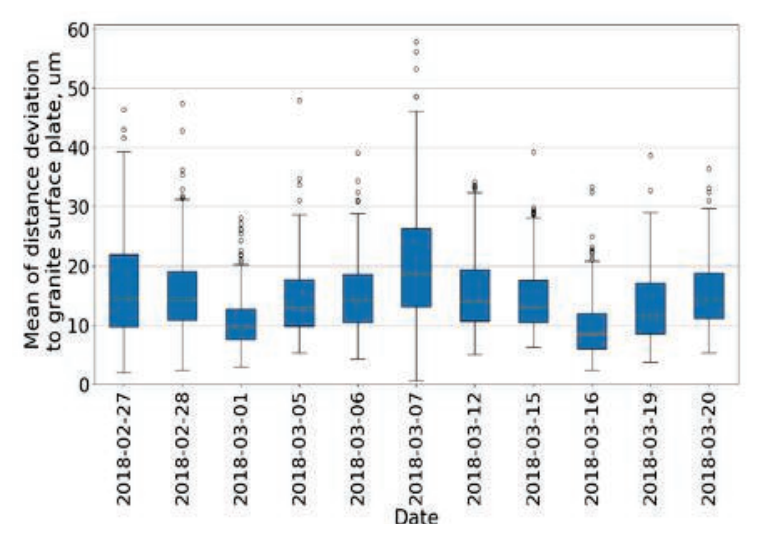

Fig. 3 Mean of distance deviation to granite surface plate

Results show us that accuracy and repeatability of measurement by the optical sensor are lower than the declared value of instrumental error of the sensor. Thus, the developed system can be successfully used to measure the surface of a deformed surface to estimate error of photogrammetric methods. 


\section{Stereo System Calibration}

To obtain reliable information about the error of the photogrammetric method, it is necessary to calibrate the cameras as accurately as possible. Another important thing is to link the object coordinates (in which the measurement is carried out by IPCT) with the coordinates in which optical sensor is moving above the surface.

The calibration process is to align points of the three-dimensional coordinate space with twodimensional points on the image. We suggest to use an optical sensor for allocating a point in space. The coordinates $x$ and $y$ of the light spot from the sensor are set in the three-dimensional coordinates (Fig. 4a) by changing optical sensor position above the surface.

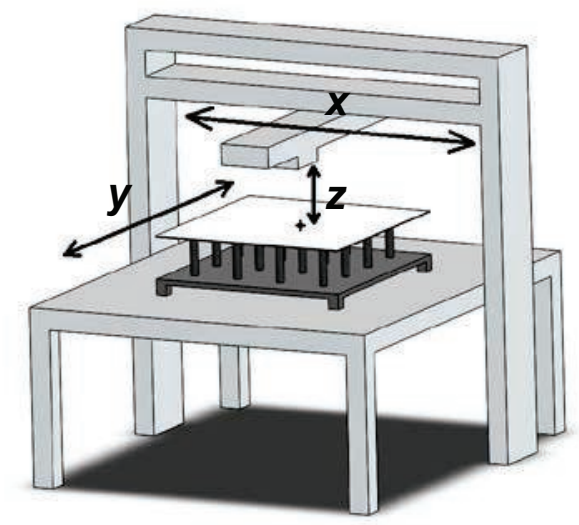

a

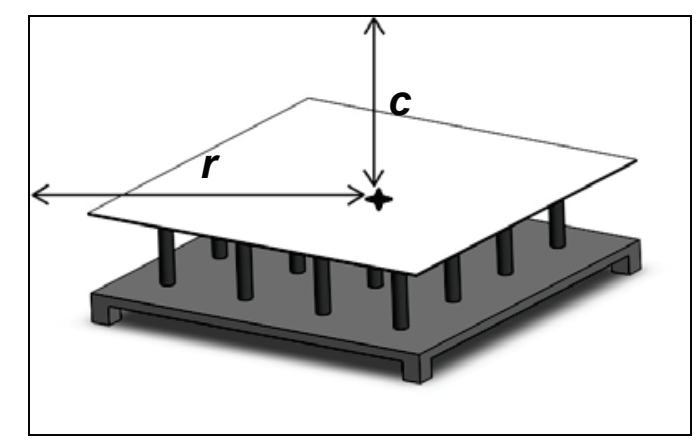

Fig. 4 Scheme of stereo system calibrating in proposed optoelectronics system:

a - scheme of determine three-dimensional coordinates; $b$ - scheme of determine twodimensional coordinates on image

The coordinate $z$ corresponds to the measured value of the distance to the surface, which can be varied with the imitator of the deformable surface. On the images obtained from the cameras (Fig. 5) we can determine the twodimensional coordinates $r$ and $c$ (Fig. 4b) by means of digital processing.

In experimental investigations a small value of the aperture diaphragm is used to increase the depth of field of the imaged space. This requires the use of additional lighting to obtain high-quality images for the IPCT. The additional illumination is not used during the calibration process which facilitates the determination of the spot light position of the optical sensor within the camera image.

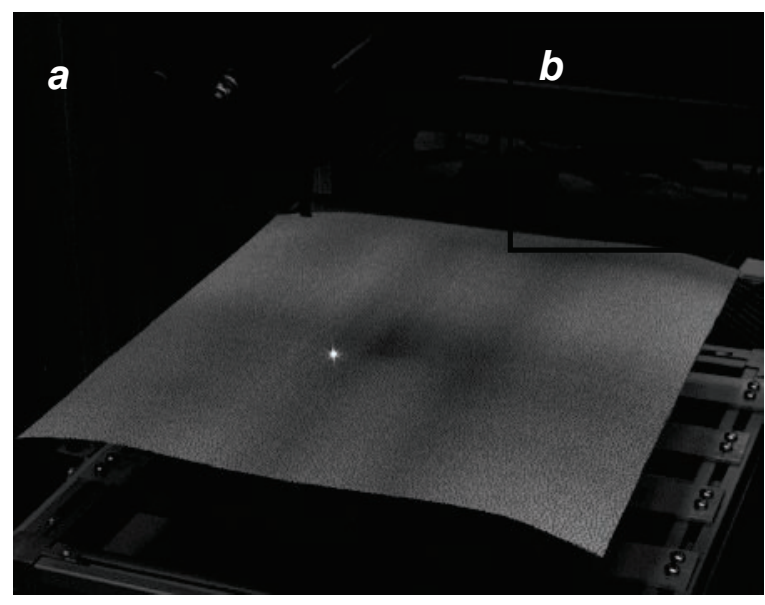

Fig. 5 Experimental image of a background pattern with a light spot:

a - image obtained by camera; $b$ - enlarged image of a light spot

The image processing searches for an associated object with total intensity greater than the specified value by means of threshold filtering to filter glares on the parts of the experimental setup. In a next step the object coordinates are calculated by center of mass method. The calibration procedure is fully automated by the software written in Python using the OpenCV library. As a calibration model the Hall method is used [16].

\section{Experimental results}

We used the following scheme of the experiment for estimating IPCT measurement error. One cycle of the experiment consists of the following steps:

1. The software randomly sets the position of the servos forming a deformation of the test surface on the imitator.

2. A stereo camera system captures images of the deformed surface and transfers them to a personal computer, where they are processed by the developed IPCT evaluation software, which delivers the surface as result.

3. The optical sensor moves by linear modules with stepper motors above the surface and measures the distance to it at several specified points.

4. The distances to the surface at these points are compared with the position of the points on the reconstructed surface profile. Thus, the desired error is estimated. 


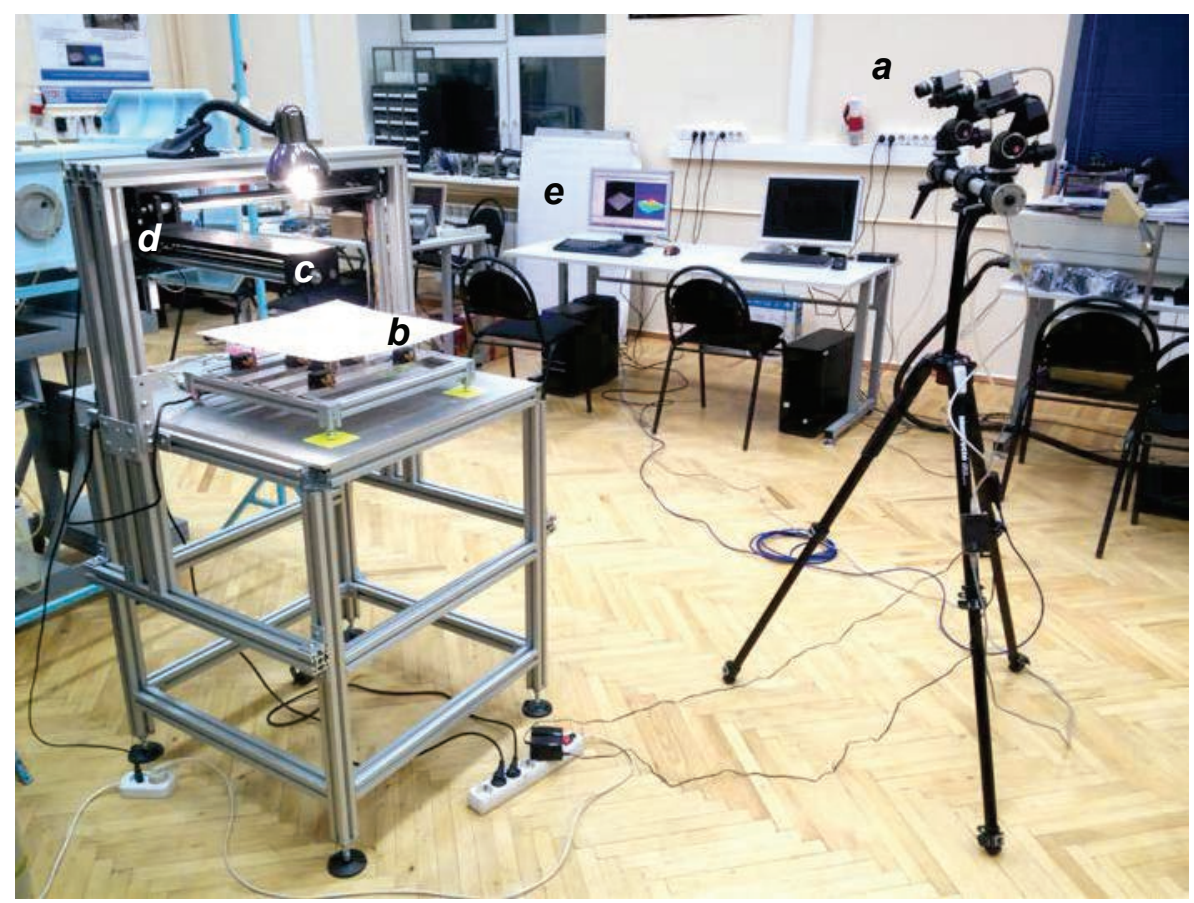

Fig. 6. The appearance of the optoelectronics system for estimating IPCT measurement error:

a - cameras stereo system; $b$ - the imitator of deformable surface; $c$ - linear modules with stepper motors; $d-$ the optical distance sensor; e - the computer with developed software

After storing the results of the steps 1-4 are repeated. The random deformation of the surface and many repeated measurements deliver a good statistical basis for the error estimation of an IPCT-setup.

In the first step the focus of the work was set to the development of the test bench consisting of the imitator of the surface deformation and an additional optical sensor. In the next step the IPCT evaluation software has to be improved. Nevertheless for the demonstration of the principle functionality of the complete system a simple IPCT evaluation routine was already implemented.

In the experimental investigation the stereo system of two Baumer VLG-24M video cameras were used. The cameras are equipped with a 1/1.2" CMOS Sony IMX249 sensor with a resolution of $1920 \times 1200$ pixels and a pixel size of $5.86 \times 5.86 \mu \mathrm{m}$. For the control and image acquisition of the cameras the Baumer API and special designed $\mathrm{C}++$ Python extension were used. The processing of the obtained images was carried out using fast Fourier transform algorithms [17].

The appearance of the system for automatic IPCT error determination is shown in Fig. 6.

In the experiment the distance from the cameras to the center of the surface was $1350 \mathrm{~mm}$. The inclination of the cameras to the surface was $20^{\circ}$. Fujinon HF25HA-1B lenses with a focal length of $25 \mathrm{~mm}$ and a relative aperture of 1:1.4 were used.

The example of the system measurement results is presented in Fig. 7. The profile of the deformed surface reconstructed by IPCT is shown in Fig. 7a. It was obtained by calculating 568 displacement vectors with a window of $128 \times 128$ pixels. The profile of the deformed surface reconstructed by optical sensor is shown in Fig. 7b. It was obtained by measuring at 121 points in $38 \mathrm{~mm}$ steps.

The difference between these two profiles (shown in Fig. 7c) is the result which allows estimating the error of the IPCT-measurement. The average value of the difference was $0.617 \mathrm{~mm}$ and its standard deviation was $0.358 \mathrm{~mm}$.

\section{Conclusions}

The paper presents an approach for estimating the measurement error for the IPCT. An experimental system is developed including an imitator of the surface deformation and an additional optical sensor for measuring the present surface profile. The error is determined as a difference between the results of the optical sensor and the IPCT. The system allows to simulate a deformation with an amplitude of $\pm 25 \mathrm{~mm}$ on a surface area of $380 \times 380 \mathrm{~mm}^{2}$ in increments of $0.5 \mathrm{~mm}$ horizontally and $0.001 \mathrm{~mm}$ vertically, with a measurement accuracy of $0.075 \mathrm{~mm}$. 
The accuracy of the IPCT is influenced by many factors like the camera calibration, the stereoscopic setup and the accuracy in the determination of point correspondences in the stereo views. The presented optoelectronic system enables a detailed investigation of different influence factors to the accuracy of IPCT for a further improvement of the method.

a

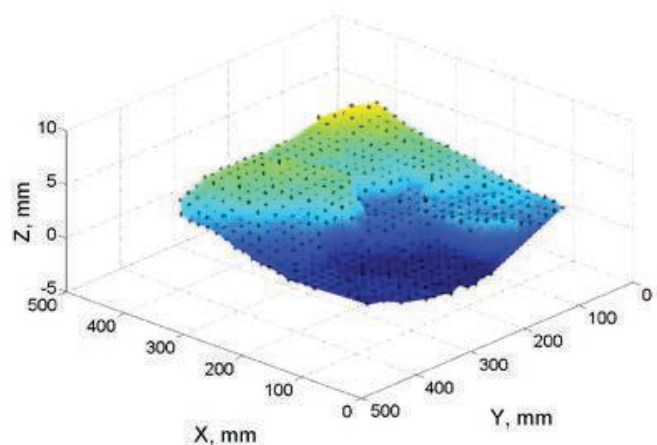

$b$

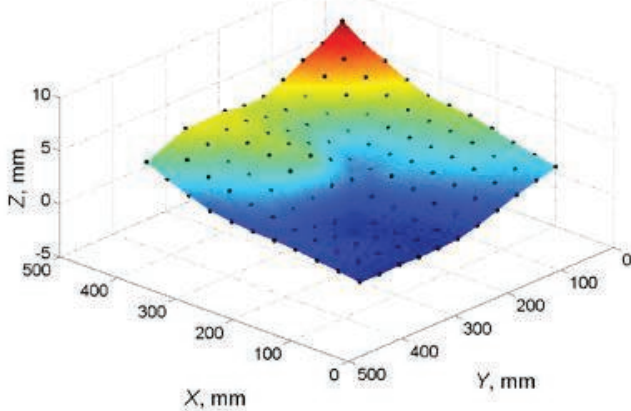

$C$

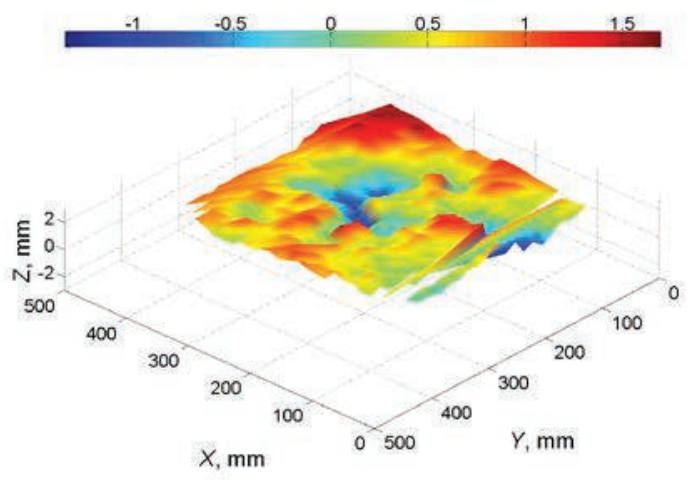

Fig. 7. The example of the system measurement results:

$a$ - The profile of the deformed surface reconstructed by IPCT; $b$ - The profile of the deformed surface reconstructed by optical sensor; $c$ - The difference between reconstructed profiles

\section{References}

[1] M. Grediac, F. Sur, B. Blaysat, The Grid Method for In-Plane Displacement and Strain Measurement: a Review and Analysis, Strain, 53(3), 205-243 (2016); doi: 10.1111/str.12182

[2] D. Tomita, M. Fujigaki, Y. Murata, Deformation Distribution Measurement from Oblique Direction Using Sampling Moire Method, Advancement of Optical Methods in Experimental Mechanics, 3, 197-204 (2015); doi: 10.1007/978-3-319-069869_22

[3] N.K. Raghuwanshi, A. Parey, Mesh Stiffness Measurement of Cracked Spur Gear by Photoelasticity Technique, Measurement, 73, 493-452 (2015); doi:

10.1016/j.measurement.2015.05.035

[4] A. Sánchez, H. Manuel, F.M. Santoyo, T. Saucedo-A, D. Reyes, Simultaneous 3D Digital Holographic Interferometry for Strain Measurements Validated with FEM, Optics and Lasers in Engineering, 52, 178-183 (2014); doi: 10.1016/j.optlaseng.2013.06.013

[5] Y. Arai, Simultaneous In-Plane and Out-of-Plane Deformation Measurement by Speckle MultiRecording Method, Measurement, 91, 582-589 (2016); doi: 10.1016/j.measurement.2016.05.037
[6] F. Boden, N. Lawson, H.W. Jentink, J. Kompenhans (Eds), Advanced In-Flight Measurement Techniques, 344 (2013); doi: 10.1007/978-3-642-34738-2

[7] R. Meyer, T. Kirmse, F. Boden, Optical In-Flight Wing Deformation Measurements with the Image Correlation Technique, New Results in Numerical and Experimental Fluid Mechanics IX, 124, 545553 (2014). Springer, Cham; doi: 10.1007/978-3319-03158-3_55

[8] A.Y. Poroikov, Reconstruction of 3D Profile of a Deformed Metallic Plate by Means of the Image Pattern Correlation Technique, Measurement Techniques, 57(4), 390-395 (2014); doi: $10.1007 / \mathrm{s} 11018-014-0466-4$

[9] B. Wieneke, PIV Uncertainty Quantification from Correlation Statistics, Measurement Science and Technology, 26 (7), 074002 (2015); doi: 10.1088/0957-0233/26/7/074002

[10] N.J. Lawson, J. Wu, Three-Dimensional Particle Image Velocimetry: Error Analysis of Stereoscopic Techniques, Measurement Science and Technology, 8(8), 894-900 (1997); doi: $10.1088 / 0957-0233 / 8 / 8 / 010$ 
[11] N.J. Lawson, J. Wu, Three-Dimensional Particle Image Velocimetry: Experimental Error Analysis of a Digital Angular Stereoscopic System,

Measurement Science and Technology, 8(12), 1455-1464 (1997); doi: 10.1088/09570233/8/12/009

[12] F. Boden, T. Kirmse, A.Yu. Poroikov, B.S. Rinkevichyus, N.M. Skornyakova, I.A. Shashkova, Accuracy of Measurement of Dynamic Surface Deformations by the Image Pattern Correlation Technique, Optoelectronics, Instrumentation and Data Processing, 50(5), 474481 (2014); doi: 10.3103/S8756699014050057

[13] V.P. Kulesh, Measurements of Deformation of the Adaptive Wing Leading Edge in a Wind Tunnel by the Videogrammetry Method, TSAGI Science Journal, 45, 993-1006 (2014); doi: 10.1615/TsAGISciJ.2015013557

[14] F. Scarano, M.L. Riethmuller, Iterative Multigrid Approach in PIV Image Processing with Discrete Window Offset, Experiments in Fluids, 26(6), 513-523 (1999); doi: 10.1007/s003480050318

[15] C. Willert, The Fully Digital Evaluation of Photographic PIV Recordings, Applied Scientific Research, 56(2-3), 79-102 (1996); doi: 10.1007/BF02249375

[16] J. Salvi, X. Armangué, J. Batlle, A Comparative Review of Camera Calibrating Methods with Accuracy Evaluation, Pattern recognition, 35(7), 1617-1635 (2002); doi: 10.1016/S00313203(01)00126-1

[17] M. Frigo, S.G. Johnson, The Design and Implementation of FFTW3, Proceedings of the IEEE, 93(2), 216-231 (2005); doi: 10.1109/JPROC.2004.840301 